\title{
Colloidal (almost) super solids
}

arXiv:1111.3466v

Authors: F. Smallenburg, L. Filion, M. Marechal, M. Dijkstra

Recommended and a commentary by Daan Frenkel, University of Cambridge

All solids at finite temperatures contain point defects such as vacancies and interstitials. Even at very low concentrations, such defects have a pronounced effect on the transport properties (e.g. electronic) of otherwise pure crystals. However, the effect of point defects on the thermodynamic properties of crystals is small, simply because the mole-fraction of defects is low. In fact, in most simple metallic and ionic crystals, but also in colloidal hard-sphere crystals, the vacancy fraction (i.e. the fraction of empty lattice sites) close to the melting transition is of the order 1 in $10^{4}$.

In a recent preprint [1], Smallenburg et al. present an astounding simulation result: some colloidal crystals may have vacancy concentrations that are almost three orders of magnitude higher than what has been observed before in simple, one-component crystals. The paper of Smallenburg et al. reports numerical simulations of the phase behavior of systems of hard, cube-shaped particles. At sufficiently high densities, such hard cubes form a simple cubic crystal. The surprising result of Ref. [1] is that these crystals contain an equilibrium concentration of vacancies that can be as high as $6 \%$. This implies that any particle will on average have more than one vacancy among its 26 neighbors. Using extensive free-energy calculations, the authors show that the very high vacancy concentration has a pronounced effect on the location of the melting transition. It should be stressed that such calculations are far from trivial because, in a finite periodic system, the number of lattice sites and the number of particles behave as independent variables $[2,3]$, yet in reality the number of vacancies is fixed by the condition that the "chemical potential" associated with vacancies must vanish (because vacancies are not conserved) [3].

Another striking feature of the calculations of Ref. [1] is that the crystallinity of the samples with vacancies is actually higher than that of a corresponding system where the number of particles is equal to the number of lattice sites. Looking at the average positions of the particles in the crystal, one observes a perfect simple cubic lattice. Yet, looking at the instantaneous snapshots of the crystal (see Fig. 1 - left), one cannot "see" vacancies: the vacancies are delocalised along rows in the crystal lattice (Fig. 1- right). This means that one cannot really speak of "vacancies" or "interstitials", only of the difference between the number of particles and the number of lattice sites. The fact that the "point" defects are actually finite "line defects" raises many interesting questions and possibilities. The first is, of course: why are vacancies delocalized? The most plausible explanation is related to the fact that cubes can form a space-filling lattice without "interdigitation": that means that entire rows of particles can slide with respect to each other without necessarily creating hard core overlaps. Yet, as Fig. 1(left) shows, the sliding of entire rows does not happen - and this is probably due to the delocalised vacancies. A vacancy can delocalize until it is blocked by a shifted row or by another delocalised vacancy. The entropic cost of 

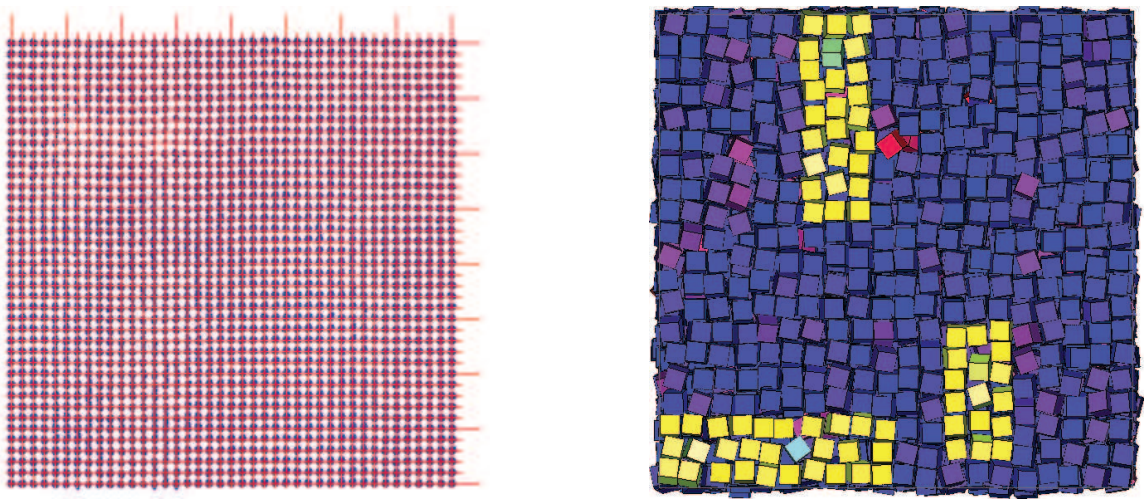

Figure 1. Left: Snapshot showing the (projected) center-of-mass positions of a system of $40^{3}$ hard cubes. Note that although the system contains $40^{3}$ particles, there are $41^{3}$ lattice sites, corresponding to a vacancy concentration of more than $7 \%$. This particular structure is mechanically stable, but the maximal equilibrium concentration of vacancies is around $6 \%$ (see Ref. [1]). Right: Snapshot of a system of hard cubes. The figure shows a single plane that contains several extended vacancies in the plane. Some of the extended vacancies have been highlighted in yellow.

delocalising an entire row would therefore be very high due to "vacancy excluded-volume" effects: delocalized vacancies behave as a polydisperse "gas" of perpendicular hard rods (similar to the well-known Zwanzig model for nematogens). One interesting question is: what determines the length of delocalized vacancy? An upper limit is set by the vacancyvacancy interaction. If we assume that line vacancies cannot intersect (and that they are not orientationally ordered) the maximum vacancy length $L$ is determined by the vacancy density $\rho$, such that $\rho L^{2}=\mathcal{O}(1)$ - but, of course, the vacancy length could be less.

The high concentration of vacancies also has a pronounced effect on the diffusivity in the crystal phase, because vacancies facilitate single-particle diffusion. But the fact that vacancies are delocalized should lead to a very interesting consequence: mass transport will be determined by the collective diffusion coefficient that should be significantly higher than the single-particle diffusion coefficient. This is easy to understand: the motion of a delocalized vacancy of length $L$ over a single lattice spacing, would correspond to the displacement of a particle over distance $L$. Hard-cube solids are clearly not "super solids", but their ability to flow whilst maintaining crystallinity should be very unusual.

Clearly, the work of ref. [1] raises many interesting questions, such as: what happens for other particles that can form space-filling structures. In fact, in a recent paper, Agarwal and Escobedo have presented a beautiful overview of the phase behavior of a wide range of space-filling, polyhedral particles [4]. This paper shows evidence for high diffusivity of hard cubes in the density regime where Ref. [1] finds a high vacancy concentration. Agarwal and Escobedo also find high diffusivity for other polyhedral hard particles systems where the possibility for "row translation" exists. However, the paper does not report collective 
diffusion constants. Refs. [1] and [4] differ in their interpretation of the phase that Ref. [1] calls simple cubic and Ref. [4] calls cubatic. Ref. [1] shows that, at least for a $40^{3}$ system, translational order extends throughout the system - and this is why a discussion in terms of a simple cubic crystal is justified. In order to know what happens in the thermodynamic limit, a full-scale finite-size scaling analysis would be required. Evidence for a bond-ordered liquid phase (a "tetratic" phase) in a system of two-dimensional hard squares was presented some time ago by Wojciechowski and Frenkel [5]. This work was subsequently extended by Donev et al. [6] to systems of hard rectangles. Ref. [5] did not explore the presence of vacancies - and yet vacancies are likely to be just as important in two dimensions as in three. Moreover vacancies make the system highly compressible and this may facilitate the formation of bond-ordered phases.

Finally, it should be stressed that crystals of hard cubes or other polyhedral particles are not purely hypothetical systems: with the recent advances in the manufacture of tailormade colloidal particles, all the phenomena discussed in the present commentary can be investigated experimentally.

\section{REFERENCES}

[1] F. Smallenburg, L. Filion, M. Marechal, M. Dijkstra, Vacancy stabilized crystalline order in hard cubes, arXiv:1111.3466v

[2] W. C. Swope and H. C. Andersen, Thermodynamics, statistical thermodynamics, and computer simulations of crystals with vacancies and interstitials, Phys. Rev. A 46, 4539(1992)

[3] B. M. Mladek,P. Charbonneau and D. Frenkel, Phase Coexistence of Cluster Crystals: Beyond the Gibbs Phase Rule, Phys. Rev. Lett. 99, 235702 (2007), B. M. Mladek, P. Charbonneau, C. N. Likos, D. Frenkel, G. Kahl, Multiple occupancy crystals formed by purely repulsive soft particles, J. Phys. Condensed Matter 20, 494245 (2008)

[4] U. Agarwal and F.A. Escobedo, Mesophase behaviour of polyhedral particles, Nature Materials 10, 230(2011).

[5] K.W. Wojciechowski and D. Frenkel, Tetratic Phase in the planar hard square system?, Comp. Meth. Sci. Tech 10, 2235-255(2004) [http://www.man.poznan.pl/cmst/www.old/vol10p2.html]

[6] A. Donev et al., Tetratic order in the phase behavior of a hard-rectangle system, Phys Rev B 73, 054109(2006). 